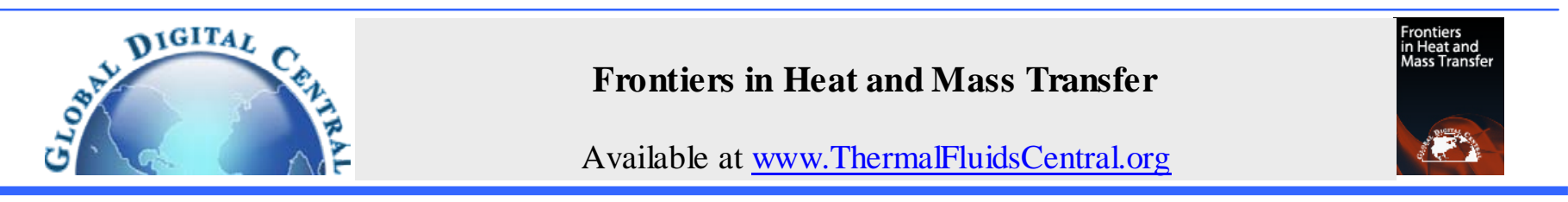

\title{
THE IMPACT OF THE RESONANCE TUBE ON PERFORMANCE OF A THERMOACOUSTIC STACK
}

\author{
Channarong Wantha ${ }^{\mathrm{a}}$, Kriengkrai Assawamartbunlue ${ }^{\mathrm{a}, *}$ \\ ${ }^{a}$ Energy Technology Research Laboratory, Department of Mechanical Engineering, \\ Faculty of Engineering, Kasetsart University, Bangkok, 10900, Thailand
}

\begin{abstract}
This paper presents a study of the impact of the resonance tube on performance of a thermoacoustic stack. The resonance tube is a key component of a standing-wave thermoacoustic refrigerator. The appropriated resonance tube's length leads to an increase of performance of the stack in terms of the temperature difference. The results also indicate that the optimal operating frequency differs from the design based on the equation of a half-wavelength. The resonance tube length is elongated to compensate for some effects that occur in the resonance tube, especially when the stack is placed in the resonance tube. The relationship of these parameters is necessary to the design and measurement performance of the thermoacoustic refrigeration system.
\end{abstract}

Keywords: Thermoacoustic; Resonance tube; Frequency; Stack

\section{INTRODUCTION}

Current research efforts on refrigeration systems are focusing on the development of alternative refrigerants and alternative technologies to reduce environmental impact, such as pulse-tube refrigeration, thermoelectric refrigeration, absorption refrigeration, etc. Thermoacoustic refrigeration is a comparatively new cooling technology, which offers a revolutionary solution for the current problems of energy consumption. Thermoacoustic refrigerators do not use refrigerant that is harmful to the environment. The refrigerant is air or other inert gases. The principle of the thermoacoustic system is based on a thermodynamics effect that occurs when a gas's temperature rises when compressed, and vice versa. The compression and expansion of gas particles occurs inside solid structures such as thermoacoustic stack (for a standing-wave thermoacoustic system). This causes heat to be transferred from one end to the other end of the stack. The result is the temperature difference generated across the stack ends. The principles of thermoacoustic systems can be found in Swift's tutorial article(1988).

A thermoacoustic refrigerator using both the travelling wave and the standing wave is notable for its simple construction and no moving parts. This is the primary benefit of the thermoacoustic refrigerator (Bassem et al., 2011; Tijani et al., 2002). It can be used in cooling electronic components, vehicles, space shuttles, and household refrigerators. However, the applications of this technology are limited by low performance of the components, such as the driver (which can produce high amplitude sound waves), the stack or the generator, and heat exchangers. Wetzel and Herman(1997) showed calculations of the coefficient of performance of the stack $\left(C O P_{s}\right)$ in a haft-wavelength of a standing-wave thermoacoustic refrigerator. They show 0.4-0.5 of Carnot's efficiency for the stack, while the coefficient of performance for the commercial refrigerator was in the range of 0.33-0.5.

There are two types of thermoacoustic refrigerator based on the source of the acoustic energy input. If the acoustic energy is provided by a driver or a commercial loudspeaker, the refrigerator is called a loudspeaker-driven thermoacoustic refrigerator. If the acoustic energy is provided by a thermoacoustic engine, the refrigerator is called thermoacoustically-driven thermoacoustic refrigerator (Babaei and Siddiqui, 2008).

The main components of a thermoacoustic refrigerator are a stack, two heat exchangers, resonance tube, and acoustic driver attached to one end of the resonance tube to generate a standing acoustic wave inside the resonance tube. Although the components of a thermoacoustic refrigerator are easily built, the designs of these components present challenges. Several authors have studied the performance of the design problem and found that the most important component of the standing-wave thermoacoustic refrigerator system is the stack. Several experimental investigations have shown that the linear thermoacoustic theory provides the optimum design of the thermoacoustic stack (Ke et al., 2010; Swift, 1988; Tijani et al., 2002). The stack is positioned between the pressure antinode and node, where an optimized temperature gradient can be generated in the stack. This temperature difference generated across the thermoacoustic stack is caused by the thermoacoustic effect (hydrodynamics heat flow). The stack material should have high heat capacity, low thermal conductivity, and an optimal value for spacing between the stack layers. Tasnim et al.,(2011) performed experiments on temperature fields at difference locations on the stack plate and in the surrounding working fluid. They found that axial heat transfer occurs at the stack extremities, as opposed to the hypothesis of a perfectly isolated stack used by Swift (1988) in the linear thermoacoustic theory. The linear thermoacoustic theory prediction is based on inviscid boundary-layer and short-stack approximation and neglecting the conduction of heat down the temperature gradient, which is small.

One reason for low performance of thermoacoustic systems is the poor performance of heat exchangers. The incorporation of the heat exchanger at both ends of the stack causes the reduction in the number of gas parcels inside the stack, resulting in a decline in system performance. Therefore, two heat exchangers (hot and cold heat exchangers) attached to both stacks must be optimum. Moreover, the design of the heat exchangers for the thermoacoustic refrigerator is challenging due to the overall heat transfer coefficient in the form of oscillation between the stack and the wall of the heat exchangers. Akhavanbazaz et al., (2007) experimentally investigated the impact of the heat exchanger area in obstructing gas flow. The results show that the gas blockage has a significant impact on the 
thermoacoustic process inside the stack. Nsofor et al., (2007) studied the convection heat transfer coefficient on the outside surface of the heat exchanger in thermoacoustic refrigeration. Results from the study showed that higher mean pressure results in a greater heat transfer coefficient if the thermoacoustic refrigerating system operates at the resonance frequency. The mean pressure and frequency also influence performance of thermoacoustic refrigerator systems (Nsofor and Ali, 2009).

As for designing the resonance tube of the thermoacoustic system, Tang et al.,(2005) and Tu et al.,(2005) found that the length of the resonance tube can significantly influence the resonance frequency; the resonance frequency decreases with the increase of the resonance tube's length. The resonance tube of the standing-wave in the thermoacoustic system can have a $\lambda / 2$ or $\lambda / 4$ straight tube length,

where $\lambda$ is the wavelength of the sound wave. That is, one end is closed and the other is connected to the acoustic driver to produce two pressure antinodes and one pressure antinod in a half-wavelength and quarter-wavelength, respectively. Setiawan et al.,(2010) used the properties of two pressure antinodes of a half-wavelength to generate a temperature difference across two stacks. Two stacks were placed at the positions near two pressure antinodes in the straight resonance tube. They found that the temperature difference occurred in both stacks inside a straight resonance tube. The optimized resonance tube in wavelength introduced by Hofler(1986) is further optimized by reducing the diameter of the resonance tube on the right of the stack using a spherical bulb to simulate an open end. Reducing the diameter in a resonance tube keeps the total loss in the resonance tube to a minimum.

The resonance frequency of the tube depends on the length of that tube and the speed of the sound. When the fluid oscillates at a half-wavelength mode, the resonance frequency of a tube may be expressed by $f_{0}=a / 2 L_{o}$. In general, this equation is applied to determine the maximum resonance tube length for the straight tube to be used with a standing-wave thermoacoustic refrigerator. Some results of the experiment found that the design parameters, such as the design of the resonance frequency of systems, are not in agreement with experimental results and affect the performance of systems. Tu et al.,(2005) showed that the stack can have an effect on the resonance frequency. Hatazawa (2006) found that the actual frequency of the thermoacoustic system is not equal to the calculation in a $\lambda / 4$ wavelength. The length of the resonance tube is extended away from the basic calculation of a $\lambda / 4$ wavelength. Assawamartbunlue and Kanjanawadee (2009) determined that the optimal operating frequency of the thermoacoustic stack is based on the peak temperature difference across the stack in a $\lambda / 4$ straight resonance tube. Setiawan et al.,(2010) calculated the resonance frequency at $f_{0}=152 \mathrm{~Hz}$ for the length of $1.12 \mathrm{~m}$ in a half-wavelength resonance tube. The results showed that with the optimum operating frequency of two stacks placed inside a resonance tube, the frequency is slightly below the calculated resonance frequency of $152 \mathrm{~Hz}$. Braun et al.,(2001) developed a half-wavelength thermoacoustic cooler prototype driven by a moving-magnet CFIC model B-300 electro-mechanical transducer. The driver was designed to deliver 300 watts at $33 \mathrm{~Hz}$. In this experiment, the leaf springs were designed to increase the mechanical resonance of the driver in order to make the combined resonance of the driver and mechanical systems occur at the desired operating frequency of around $170 \mathrm{~Hz}$. Tijani (2001) showed that the electroacoustic efficiency (defined as the output acoustic power divided by the input electrical power) can be maximized by matching the resonance frequency of the driver to the resonance frequency of the resonance tube, and the resonance frequency of the driver can be shifted to match the resonance frequency of the resonance tube by adjusting the volume of gas at the back of the driver housing.

The objective of this paper is to investigate the impact of the resonance tube on the performance of the thermoacoustic stack. A standing-wave thermoacoustic refrigerator prototype was assembled for experimentation within such parameters. In order to understand the effect of the resonance tube on the performance of the thermoacoustic stack, the hot and cold heat exchangers were removed from the experiment. Results from study assist in determining the optimum design of the straight resonance tube in a half-wavelength of the thermoacoustic refrigerator.

\section{DES IGN OF THE THERMOACOUS TIC STACK}

The performance of the thermoacoustic system depends on the porous medium used to exchange heat with the working fluid in a stack or a regenerator. The term regenerator is used when the pore size of the porous element is small compared to a thermal penetration depth. The thermal penetration depth is the layer around the stack plate where the thermoacoustic phenomenon occurs. Conversely, it is called the stack, when this porous medium is loosely packed compared to a thermal penetration depth.

The thermoacoustic effects occur in the stack through the interaction of sound waves and the stack plate. As a gas parcel moves toward the pressure antinode, the temperature of the gas is increased because of acoustic compression and the excess heat is transferred to the stack plate. When a gas parcel moves back to the pressure node and expands, the temperature of the gas parcel reduces lower than the stack plate. The gas parcel gains heat from the plate and starts the cycle again. Detailed explanation of the thermoacoustic effect can be obtained from other sources (Swift, 1988; Tijani, 2001; Wetzel and Herman, 1997).

To develop a standing-wave thermoacoustic refrigerator, the basic equation and theory for linear thermoacoustic systems is well covered by Swift (1988) and Tijani et al.,(2002). The thermal and viscous penetration depths are given by:

$$
\delta_{\kappa}=\sqrt{\frac{2 K}{\rho c_{p} \omega}}
$$

and

$$
\delta_{v}=\sqrt{\frac{2 \mu}{\rho \omega}}
$$

Where $\omega=2 \pi f_{0}$ is the angular frequency of the sound wave, $f_{0}$ is the design frequency, and $K$ is the thermal conductivity. Here, $\mu, \rho$ and $c_{p}$ are the viscosity, density, and isobaric specific heat of the gas, respectively.

The performance of the thermoacoustic stack depends on three main stack design parameters: the center position, $X_{s n}$, the length, $L_{s n}$, and the cross-section area, $A$. The normalized cooling power, $Q_{c n}=Q_{c} / P_{m} A$, and acoustic power, $W_{n}=W / P_{m} a A$, are given by Tijani et al., (2002):

$$
\begin{aligned}
Q_{c n}= & -\left[\frac{\delta_{\kappa n} D^{2} \sin \left(2 X_{s n}\right)}{8 \gamma(1+\sigma)\left(1-\sqrt{\sigma} \delta_{\kappa n}+\frac{1}{2} \sigma \delta_{\kappa n}^{2}\right)}\right] \\
& \times\left[\frac{\Delta T_{m n} \tan \left(X_{s n}\right)}{(\gamma-1) B L_{s n}} \times \frac{(1+\sqrt{\sigma}+\sigma)}{1+\sqrt{\sigma}}-\left(1+\sqrt{\sigma}-\sqrt{\sigma} \delta_{\kappa n}\right)\right]
\end{aligned}
$$

and

$$
\begin{aligned}
W_{n}= & {\left[\frac{\delta_{\kappa n} D^{2} L_{s n}(\gamma-1) B \cos ^{2}\left(X_{s n}\right)}{4 \gamma}\right] } \\
& \times\left(\frac{\Delta T_{m n} \tan \left(X_{s n}\right)}{B L_{s n}(\gamma-1)(1+\sqrt{\sigma})\left(1-\sqrt{\sigma} \delta_{\kappa n}+\frac{1}{2} \sigma \delta_{\kappa n}^{2}\right)}-1\right)
\end{aligned}
$$




$$
-\left(\frac{\delta_{\kappa n} L_{s n} D^{2}}{4 \gamma} \times \frac{\sqrt{\sigma} \sin ^{2}\left(X_{s n}\right)}{B\left(1-\sqrt{\sigma} \delta_{\kappa n}+\frac{1}{2} \sigma \delta_{\kappa n}^{2}\right)}\right)
$$

Where $\delta_{\kappa n}=\delta_{\kappa} / y_{o}$ is the normalized thermal penetration depth, $B=y_{o} /\left(y_{o}+l\right)$ is the blockage ratio of the stack, $y_{o}$ is half of the distance between the stack layers, $l$ is half of the thickness of the stack layers, $\Delta T_{m n}=\Delta T_{m} / T_{m}$ is the normalized temperature difference, and $D=P_{o} / P_{m}$ is the drive ratio. The design parameters used in the experiments are listed in Table 1.

As shown in Eq.(1) through Eq.(4), the design of the thermoacoustic stack was affected by several parameters and is a function of the resonance frequency. However, some of these parameters are constant, such as the blockage ratio, fluid properties, and some geometrical parameters of the stack. Equation (1) indicates that the design frequency must not be too large because the spacing in the stack is a function of $\delta_{\kappa}$. A high frequency requires very small spacing, which is difficult to manufacture. Therefore, the frequency of $f_{0}=440 \mathrm{~Hz}$ is selected as the design frequency. Since the stack material should have good heat capacity and low thermal conductivity, the spiral stack was made from a $1.2 \times 10^{-4} \mathrm{~m}$ thick Mylar sheet and each layer of rolled plate was divided by fishing lines. The gaps between the layers are used to estimate the parallel plate channels; plate spacing is around $2 y_{o}$. The stack performance depends on the blockage ratio and the length, which can be optimized by optimizing the coefficient of performance of the $\operatorname{stack}\left(C O P_{s}=Q_{c n} / W_{n}\right)$.

In order to find the impact of the resonance tubes on the temperature difference across the stack, the stack length was kept equal to $L_{s n}=0.24$. Also, the blockage ratio of the stack was kept constant. This led to the optimum position of each stack plated in the resonance tube. However, there are some conditions to consider if the stack is changed with design frequency, i.e. $\delta_{\kappa}$ and $\delta_{v}$.

In the $\mathrm{COP}_{s}$ calculation, Eqs.(3) and (4) are used. To increase the efficiency of the stack, the stack must be fixed between the pressure antinode and pressure node and have a maximum difference in pressure amplitude. The positions of the pressure antinode and node in the tube depend on the frequency in the tube. In order to determine the optimum stack position inside the resonance tube, the normalized stack position $X_{s n}$ was changed from 0.26 to 0.43 . Typically, the $C O P_{s}$ increases when the normalized stack position is decreased. That is, the stack was placed close to the pressure antinode or the velocity node (for a half-wavelength tube). Tijani et al., (2002) described that viscous losses in Eq.(4) are proportional to the square of the acoustic velocity. Thus, decreasing the velocity will result in a decrease of loss and increase the $\mathrm{COP}_{s}$.

Conversely, if the normalized stack position was shifted from the position with maximum pressure amplitude, it will result in increases to both the losses and the normalized stack length (see Fig.1). Figure 2 shows that when the stack length was kept at $L_{s n}=0.24$, and as the stack was placed closer to the driver or the pressure antinode, the $C O P_{s}$ decreased. It can be concluded that when normalized stack length is kept at a constant value during the calculation, the $C O P_{\mathrm{s}}$ changed with normalized stack position, $X_{\mathrm{sn}}$.

Figure 2 shows that the $C O P_{s}$ peak was $X_{s n}=0.26$.

In this experiment, the loudspeaker was used as the acoustic driver. To avoid the possibility of heat transfer from the voice coil of the loudspeaker to the stack end, the position of the stack inside the resonance tube was shifted from the loudspeaker. This means that the $C O P_{s}$ decreased while increasing the normalized stack position. Based on the theoretical results and for practical reasons, we decided to set $X_{s n}=0.39$ as the position of the stack placed inside the resonance tube. Therefore, the calculated values of the stack position at $X_{s n}=0.39$ are used in further experiments.

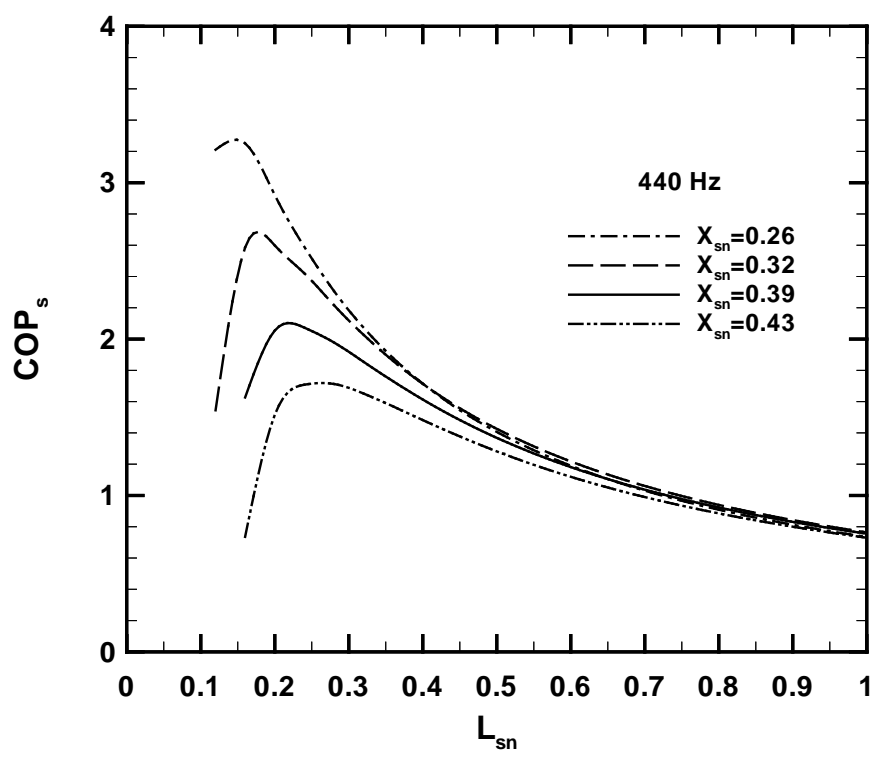

Fig. 1 The $C O P_{s}$ versus the normalized stack length for different values of the normalized stack position.

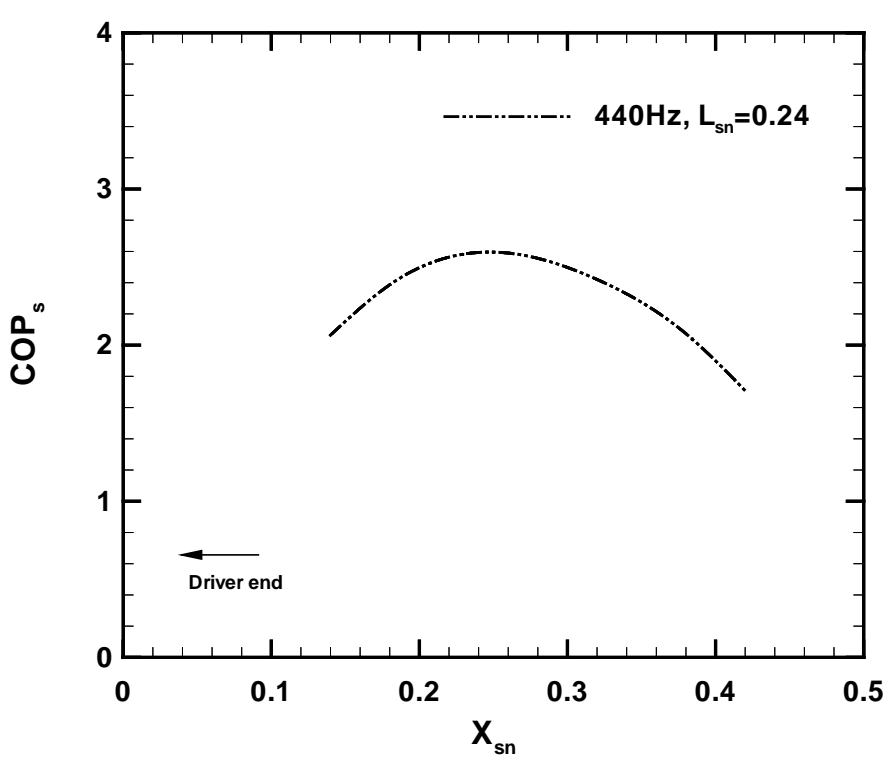

Fig. 2 The $C O P_{s}$ versus the normalized stack positions.

\section{EXPERIMENTAL APPARATUS AND PROCEDURE}

The schematic of a standing-wave thermoacoustic refrigerator is shown in Fig. 3(a) and (b), which is placed horizontally. A four-inch diameter loudspeaker with $8 \mathrm{ohm}$ and the cone volume of $26.507 \mathrm{~m}^{3}$ $\left(V_{c}=\pi r^{2} h / 3\right)$ was used as the acoustic driver. The driver was driven by a function generator and a power amplifier was used to provide the required voltage and adjust the operating frequency. The input voltage to the loudspeaker remained constant at $10.1 \mathrm{~V}$ during the experiments. The driver was mounted in a plastic housing (an enclosure) to which the resonance tube was connected. In the present study, the volume of air at the back of the driver housing was constant at a ratio of $V_{b} / V_{c}=24.52$. It is called the back volume $V_{b}$ (see Fig. 3(b)). In order to avoid heat loss from 

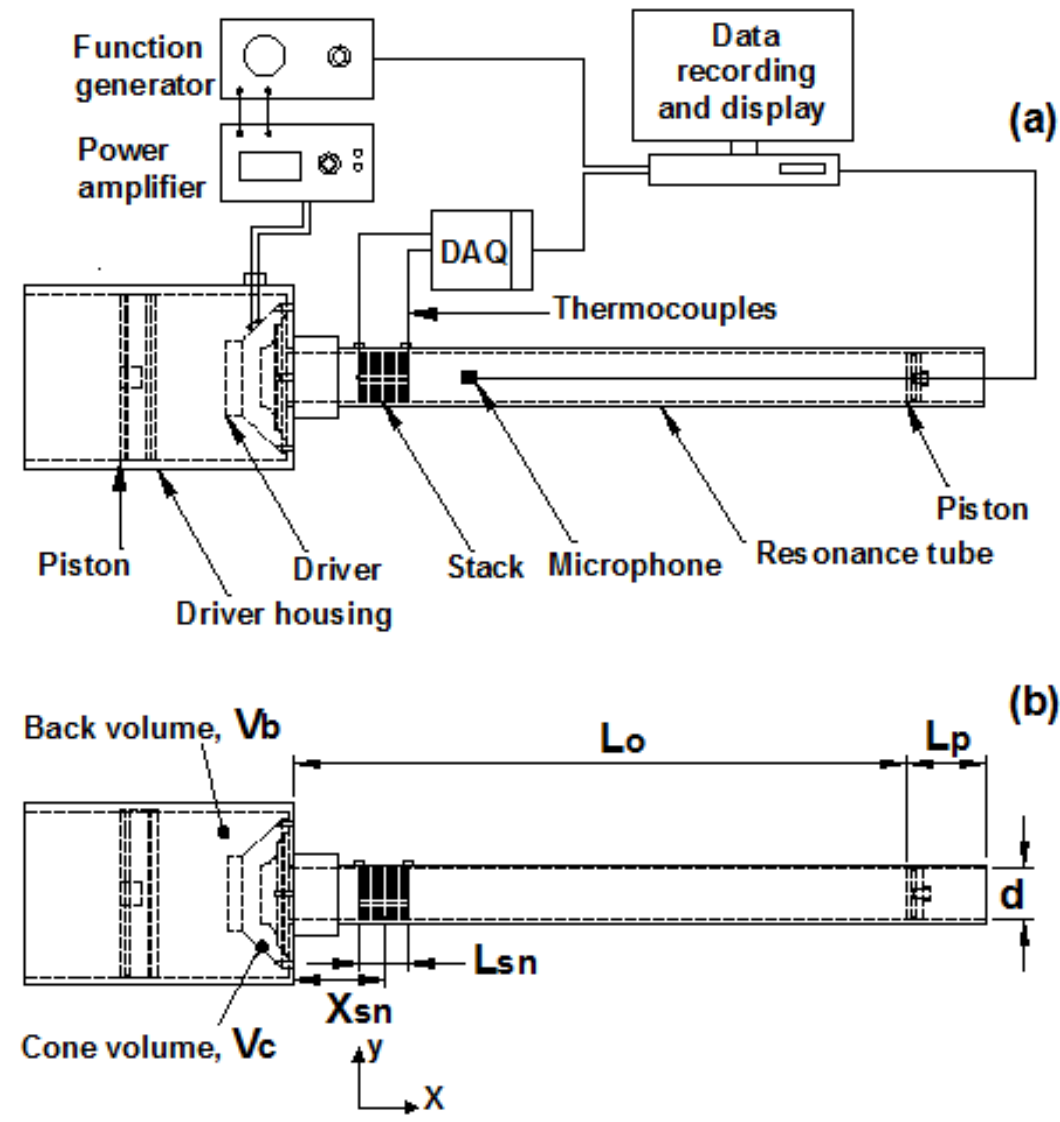

(b)

Fig. 3 A schematic of the standing-wave thermoacoustic refrigerator: (a) setup used to measure the temperature and the acoustic pressure distribution, (b) experimental parameters for resonance tube measurements.

the resonance tube, which is proportional to the surface area of the resonance tube, the resonance tube was a straight acrylic tube. Its internal diameter was $d=0.034 \mathrm{~m}$, and the material was $3 \times 10^{-3} \mathrm{~m}$ thick. To be able to adjust the length of the resonance tube so as to change the operating frequency of the tube, a movable piston was placed inside the resonance tube (see Fig.3 (a)). The half-wavelength of the standing wave is defined as

$$
f_{0}=\frac{a}{2 L_{o}}
$$

Where $f_{0}$ and $L_{o}$ are the resonance frequency and the length of the resonance tube, respectively. Equation (5) is the general equation for the fundamental resonance frequency of a tube. This frequency is usually used as the operating frequency for the produced thermoacoustic effect. The speed of sound in air is defined as

$$
a=\sqrt{\gamma R T_{m}}
$$

Where $R$ and $T_{m}$ are the gas constant and the mean fluid temperature, respectively.

The length of the resonance tube of the frequency $440 \mathrm{~Hz}$ corresponding to Eqs.(5) and (6) is $L_{o}=0.395 \mathrm{~m}$, which is the measurement from the front of the driver to the end of the straight acrylic tube, as shown in Fig.3(b). The acoustic pressure and sound intensity level were measured using a condenser microphone $(5 \mathrm{mV} / \mathrm{Pa}$ of sensitivity). To measure the pressure distribution along the resonant tube, a microphone was freely positioned inside the resonance tube and a personal computer was used for the data display. In the linear thermoacoustics theory, the thermoacoustic effects occur inside the stack (Swift, 1988). This is reliable at low acoustic pressure amplitude. However, Tasnim et al.,(2011) found that axial heat transfer occurs at stack extremities. In the case of a high acoustic pressure level inside the resonance tube, nonlinearities appear and become more important (Berson et al., 2008). Thus, for a thermoacoustic refrigerator, no thermal reservoirs were connected to the stack ends and the thermoacoustic heat flow was sustained from the plates themselves; therefore, heat flows from the cold gas to the cold stack end. Conversely, if the gas temperature is lower than the hot stack end temperature, the heat will flow from the hot stack end to the cold gas (Tasnim et al., 2011). Thus, the temperature differences generated across the stack were measured by two K-type thermocouples were placed in contact with the left and right ends of the stack as shown in Fig.3 (a). The accuracy of the thermocouple was $\pm 0.2^{\circ} \mathrm{C}$.The OMB-DAQ BOARD-3000 via an OMB-PDQ30 and DAQVIEW software by Omega Engineering were used to acquire the temperature data. Three experimental runs were conducted for each set of experiments to verify consistency. The experiment uses air as the working fluid at the atmospheric pressure.

\section{EXPERIMENTAL RES ULTS AND DIS CUSSION}

The characteristic of sound waves inside a half-wavelength resonance tube was investigated. From Eq.(5), when the frequency is $f_{0}=440 \mathrm{~Hz}$, the length of the resonance tube was set equal to $L_{o}=0.395 \mathrm{~m}$. To verify that the pressure antinode and node corresponded with the frequency of $440 \mathrm{~Hz}$, the pressure distribution along the axis in the resonance tube was measured using a condenser microphone. However, this experiment was carried out without incorporating the stack.

Figure 4 shows that the minimum pressure exists at the middle of the resonance tube and the maximum pressure is in front of the driver. This indicates that characteristics of the sound wave in the resonance tube corresp ond to a half-wavelength of the standing wave, which agrees with the calculation in Eq.(5). As discussed above, to enhance the efficiency of the stack, the stack must be placed between the pressure antinode and pressure node and must have a maximum difference in pressure amplitude. From the results of the calculation for the stack in Fig. 2, the optimum position of the 
Table 1 The design parameters for the present study.

\begin{tabular}{|l|c|l|}
\hline \multicolumn{1}{|c|}{ Property or parameter } & Symbol & \multicolumn{1}{c|}{ Value } \\
\hline Speed of sound in gas & $a$ & $347.2 \mathrm{~m} / \mathrm{s}$ \\
\hline Ratio of specific heat & $\gamma$ & 1.4 \\
\hline Mean temperature & $T_{m}$ & $300 \mathrm{~K}$ \\
\hline Gas Prandtl number & $\sigma$ & 0.7 \\
\hline Mean pressure & $P_{m}$ & $101.3 \mathrm{kPa}$ \\
\hline Frequency & $f_{0}$ & $440 \mathrm{~Hz}$ \\
\hline Blockage ratio & $B$ & 0.744 \\
\hline Drive ratio & $D$ & 0.013 \\
\hline Wave number & $k$ & $7.238 \mathrm{~m}^{-1}$ \\
\hline Thermal penetration depth & $\delta_{\kappa}$ & $1.266 \times 10^{-4} \mathrm{~m}$ \\
\hline Viscous penetration depth & $\delta_{v}$ & $1.064 \times 10^{-4} \mathrm{~m}$ \\
\hline Temperature difference & $\Delta T_{m}$ & $25 \mathrm{~K}$ \\
\hline $\begin{array}{l}\text { Normalized stack position } \\
\text { (ref. from the driver) }\end{array}$ & $X_{s n}=k x_{s}$ & 0.24 \\
\hline Normalized stack length & $L_{s n}=k L_{s}$ & 0.39 \\
\hline $\begin{array}{l}\text { Cone volume of a } \\
\text { loudspeaker }\end{array}$ & $V_{c}$ & $26.507 \mathrm{~m}^{3}$ \\
\hline
\end{tabular}

stack is $X_{s n}=0.39$, or $x_{s}=0.054 \mathrm{~m}$. The results in Fig.4 show that at this stack position, the stack will be placed between the pressure antinode and the pressure node of the resonance tube's length of $L_{o}=0.395 \mathrm{~m}$.

Frequency is vital to thermoacoustic systems. Since the frequency can create the thermoacoustic effect inside the stack, the resonance frequency was used as the operating frequency to run the system. In order to check that the optimum operating frequency of the system is $440 \mathrm{~Hz}$, two methods can be used: determination of the amplitude of the pressure compared to the frequency or determining the temperature difference across the stack compared to the frequency. In this experiment, the temperature compared to the operating frequency was determined to find the optimum operating frequency.

Figure 5 shows the temperature difference across the stack. During the experiments, the power input was set equal to $10.1 \mathrm{~V}$ and the stack position remained at its optimal position. The results showed that the operating frequency equaled the design resonance frequency at $f_{0}=440 \mathrm{~Hz}$ and the resonance tube's length was kept at $L_{o}=0.395 \mathrm{~m}$ or $L_{p} / L_{o}=0 \%$, where $L_{p}$ is the length of the resonance tube increased from $L_{o}$. The temperature difference across the stack ends was as high as $12.5^{\circ} \mathrm{C}$ (see solid line inFig.5). It can be seen that the experimentally obtained temperature difference value does not come close to the design temperature difference $\left(\Delta T_{m}\right)$, as shown in Table 1. However, when the operating frequency is further increases, the temperature difference across the stack ends increases continuously with operating frequency. As the operating frequency increases from $440 \mathrm{~Hz}$ to $480 \mathrm{~Hz}$, the temperature increases from $12.5^{\circ} \mathrm{C}$ to $26^{\circ} \mathrm{C}$; however, when further variation in the operating frequency exceeded $480 \mathrm{~Hz}$, a decrease in temperature difference across the stack was noted (see solid line in Fig.5). Due to the influence of the thermal penetration depth inside the stack, a high operating frequency leads to low thermal penetration depth. It will also increase the viscous loss and abate the thermoacoustic effects. Thus, further changes in the frequency resulted in a decrease in the temperature difference across the stack, as shown at $500 \mathrm{~Hz}$ in Fig.5.

Also, Fig. 5 shows that the maximum value of the temperature difference occurs at the operating frequency of $480 \mathrm{~Hz}$. The temperature difference is increased by approximately $52 \%$ compared with $f_{0}=440 \mathrm{~Hz}$, at $L_{p} / L_{o}=0 \%$. It is concluded that under the resonance tube's length of $L_{p} / L_{o}=0 \%$ or $L_{o}=0.395$, the

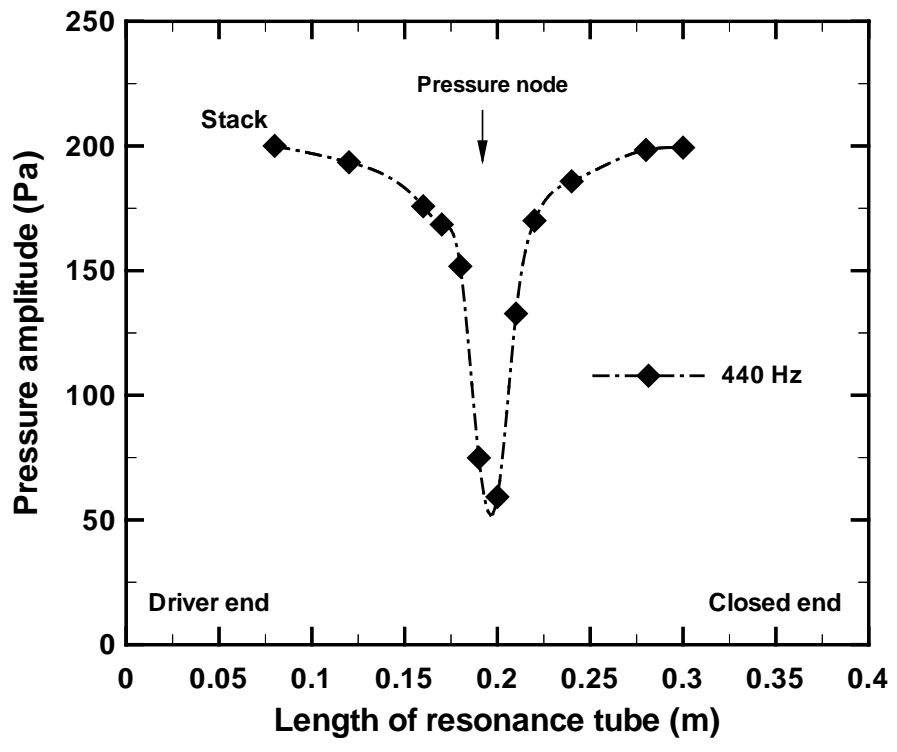

Fig. 4 The pressure distribution inside the resonance tube.

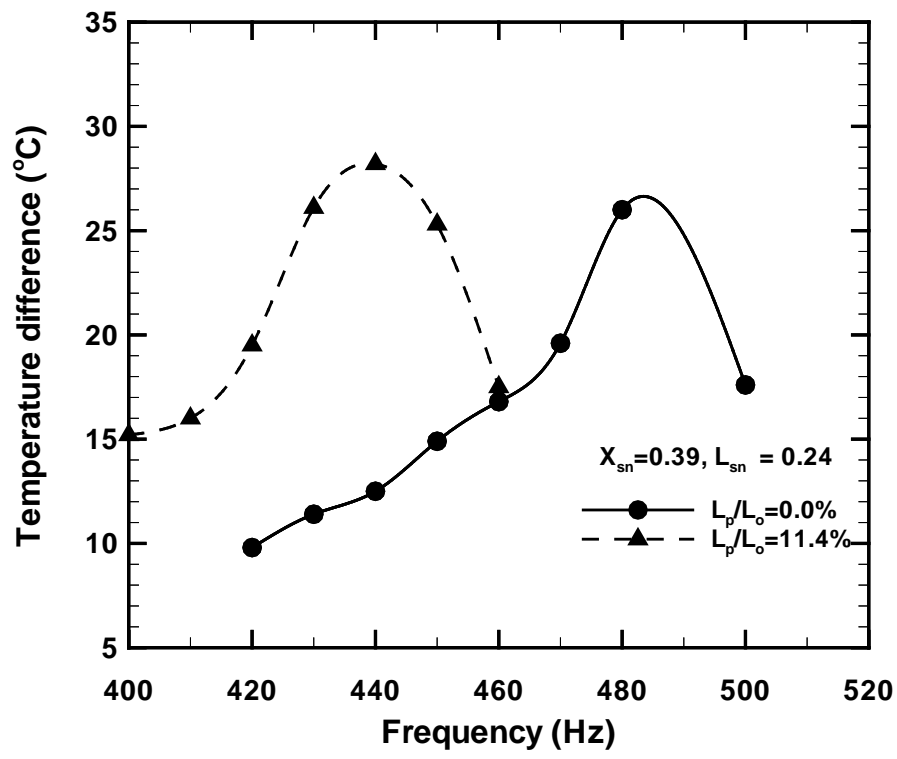

Fig. 5 The effect of the operating frequency on the temperature difference across the stack.

optimal operating frequency of the system is $480 \mathrm{~Hz}$. The temperature difference that occurs at $480 \mathrm{~Hz}$ agrees with the $\Delta T_{m}$ used for the design of the stack, as shown in Table 1. However, the experiment's results indicate that the frequency of $480 \mathrm{~Hz}$ is not equal to the calculated frequency in Eq.(5), where in the length of the resonance tube is $L_{o}=0.395 \mathrm{~m}$.

The reason for the difference between the experimental and theoretical results might be due to the behavior of the sound wave reflecting at an open end. It shows that this does not occur completely at the end section of the resonance tube, but a small distance outside the resonance tube. In addition, the volume of the air at the back of the loudspeaker housing has a significant influence on the resonance frequency of the system because the back volume of the driver housing is related to the mechanical compliance and the resonance frequency of the loudspeaker(Beranek, 1954). However, the volume of the air at the back of the loudspeaker housing is limited by the size of the loudspeaker. The final effect on the frequency is some effect from the stack. Note that when the stack is placed inside the resonance tube of $L_{o}=0.395 \mathrm{~m}$ and measured in terms of the temperature difference, the optimal operating frequency is shifted away from the expected value of $440 \mathrm{~Hz}$ to $480 \mathrm{~Hz}$ to compensate for 


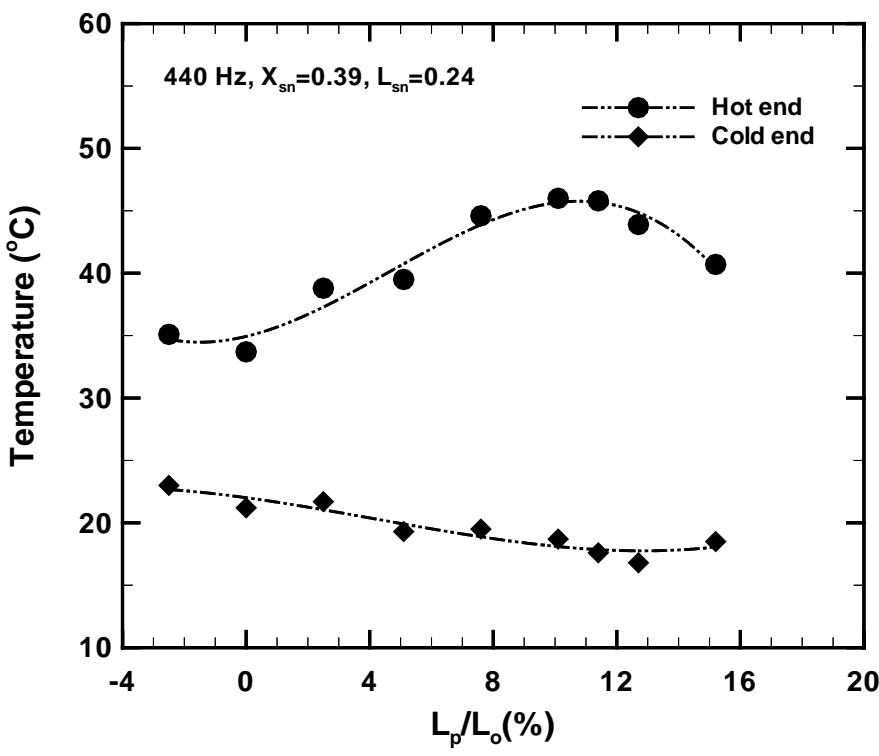

Fig. 6 The effect of the resonance tube length on the temperature of the stack.

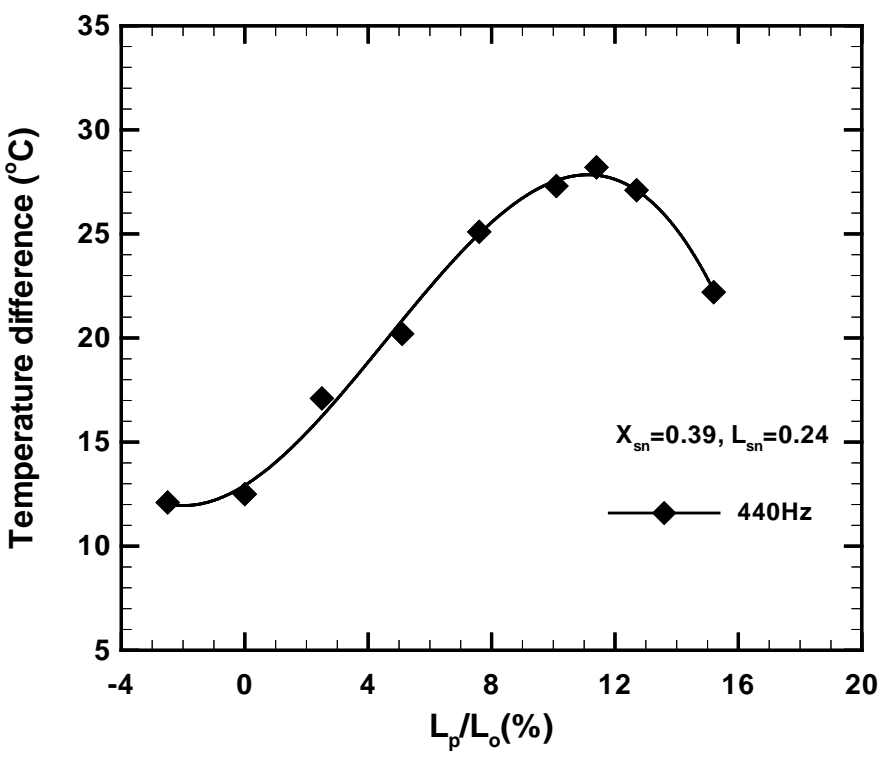

Fig. 7 The effect of the resonance tube length on the temperature difference across the stack .

the same effect discussed above. Thus, the stack can influence the resonance frequency of the thermoacoustic system.

Even though the frequency of $480 \mathrm{~Hz}$ was able to produce the thermoacoustic phenomenon through the stack, the study might not reflect optimal conditions to produce high performance for this stack. When a gas parcel moves to the layers of stack plates, the influence of the thermal and viscous penetration depth become more important. Thus, the optimal operating frequency of the system ought to correspond with the values of the thermal and viscous penetration depth used for the design conditions.

The next results are obtained when decreasing the frequency of $480 \mathrm{~Hz}$ to the expected value of the frequency at $440 \mathrm{~Hz}$. Since the fundamental resonance frequency of a tube is a function of the sound velocity and length of that tube, changing the length of the resonance tube will lead to either an increase or decrease of the resonance frequency of that tube. Thus, we studied the impact of the resonance tube with difference lengths on the resonance frequency of the thermoacoustic stack; an experiment was carried out in terms of the temperature across the stack ends. The length of the resonance tube is changed by the movable piston attached at the end of the resonance tube, as shown in Fig.3 (a). The operating frequency from the function generator is kept at the frequency of $440 \mathrm{~Hz}$, which corresponds to the design targets of the stack in Table 1.

Figure 6 shows the relationship of the temperature of the stack ends to the resonance tube's length, which varied from $L_{p} / L_{o}=0 \%$ to $L_{p} / L_{o}=15.2 \%$ with around a $2.5 \%$ increment. It can be seen that when the resonance tube is elon gated from $L_{p} / L_{o}=0 \%$ (unchanged), the cold end of the stack is incrementally decreased. From this result, the cold side of the stack had a temperature as low as $16.8{ }^{\circ} \mathrm{C}$ at $L_{p} / L_{o}=12.7 \%$. Meanwhile, the opposite end of the stack markedly rose with an increase in the length of the resonance tube. However, when further changes in the resonance tube's length were made, the temperature of the hotter side of the stack dropped and tended to move back to the temperature in the initial conditions.

The impact of the resonance tube's length in terms of the temperature difference across the stack is shown in Fig.7. Typically, performance of the stack depends upon the drive ratio, the Prant number of working fluid, the operating frequency, the property of material, and the pore size of the stack. In addition to those factors, our studies have shown that the performance of the stack is a function of the resonance tube's length. As the resonance tube's length increased from $L_{p} / L_{o}=0.0 \%$ to $L_{p} / L_{o}=11.4 \%$, the temperature difference across the stack increased by approximately $55.67 \%$ compared with the case of $L_{p} / L_{o}=0.0 \%$ at $440 \mathrm{~Hz}$ (see Fig.7). Similar to what is shown in Fig.6, this trend stops at a certain length (see Fig.7). Note that the efficiency of a thermoacoustic stack might be changed by the absence of two heat exchangers.

As discussed above, to enhance the performance of the stack in thermoacoustic systems, the thermal penetration depth, viscous loss, and spacing of the stack in the form of the blockage ratio should correspond to the operating frequency. To further verify the conclusion, Fig.5 (dashed line) shows the results of the experiments of the length of the resonance tube compared to the operating frequency. The experiment indicates that the operating frequency at $480 \mathrm{~Hz}$ decreases to $440 \mathrm{~Hz}$ with an increase of the resonance tube's length at $L_{p} / L_{o}=11.4 \%$. Therefore, a high temperature difference across the stack depends on the optimal resonance tube's length matching the operating frequency of the stack. From Fig. 5, it can be concluded that with the length more or less at $L_{p} / L_{o}=11.4 \%$, the frequency changed and led to the decreases in the temperature differences across the stack, as shown in Fig.6 and Fig.7.

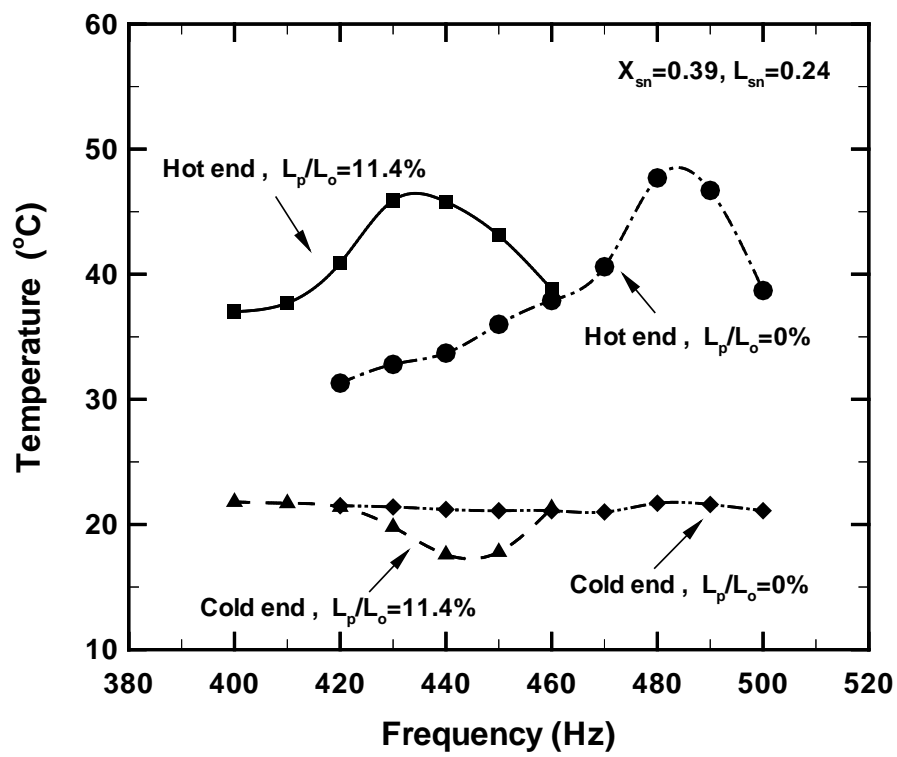

Fig. 8 The hot and cold temperature of the stack ends versus the op erating frequency. 
Figure 8 shows the temperature of the hot side and the cold side of the stack end compared to operating frequency. The temperature was compared between the two lengths of the resonance tube at $L_{p} / L_{o}=11.4 \%$ and $L_{p} / L_{o}=0 \%$. As the operating frequency increases from $400 \mathrm{~Hz}$ to $500 \mathrm{~Hz}$, the length of the resonance tube is kept at $L_{p} / L_{o}=0 \%$. This did not significantly enhance the temperature of the cold side of the stack; however, the temperature of the cold end stack changed consistently with increases in operating frequency. The hot end temperature markedly rose with the change in operating frequency. When $L_{p} / L_{o}=11.4 \%$ and the operating frequency was increased from $430 \mathrm{~Hz}$ to $450 \mathrm{~Hz}$, the hot end of the stack had the highest temperature and the cold end of the stack has the lowest temperature inside the resonance tube. It can be concluded that when the length of the resonance tube matches the operating frequency of the system (i.e., when the thermal penetration depth, viscous loss, and spacing of the stack in the form of the blockage ratio corresponded to the operating frequency of the stack design conditions, which is at $440 \mathrm{~Hz}, L_{p} / L_{o}=11.4 \%$ ), the temperature difference across the stack increased by approximately $7.8 \%$ compared to the results at $480 \mathrm{~Hz}$, or $L_{p} / L_{o}=0 \%$ (see the solid line and dashed line in Fig.5).

It indicates that the length of the resonance tube plays a significant role in the frequency and performance of the thermoacoustic stack and confirms that the length of the resonance tube, as calculated by Eq.(5), requires compensation to match the operating frequency and the design frequency of the stack. For this work, the corrected tube length of a $\lambda / 2$ wavelength with incorporating the stack is $L_{p} / L_{o}=11.4 \%$. For this adjustment, the resonance frequency in the tube must correspond to the frequency of design of the stack to maintain the thermal and viscous penetration depth and improve the performance of the thermoacoustic stack. The length of the resonance tube as presented in Eq.(5) must be increased by 0.114 times the length, $L_{o}$.

\section{CONCLUSION}

The impact of the resonance tube on a thermoacoustic refrigerator in terms of the temperature difference across the stack was studied. The resonance tube is a key component of a thermoacoustic refrigerator. Both the operating frequency and the resonance tube's length play an important role in stack performance. The appropriated resonance tube's length leads to an increase in performance of the stack in terms of the temperature difference. However, a poor match between the operating frequency and the length of the resonance tube will cause a decline in stack performance. The results also indicate that the optimal operating frequency differs from the design based on the equation $f_{0}=a / 2 L_{o}$. The resonance tube length is elongated to compensate for effects that occur in the resonance tube (i.e., the stack is placed in the resonance tube). When the optimal combination of the operating frequency and the resonance tube's length is achieved, the temperature difference is increased by approximately $55.67 \%$. The relationship between these parameters is essential to the design and measurement performance of thermoacoustic refrigeration systems.

\section{ACKNOWLEDGEMENTS}

The research is financially supported by the Kasetsart University Research and Development Institute, Bangkok, Thailand.

\section{NOMENCLATURE}

cross-section area of the stack, $\mathrm{m}^{2}$

speed of sound $(\mathrm{m} / \mathrm{s})$

blockage ratio

C

isobaric specific heat $(\mathrm{J} / \mathrm{kg} \cdot \mathrm{K})$
$\mathrm{COP}_{\mathrm{s}}$ coefficient of performance of the stack

$c_{v} \quad$ isochoric specific heat $(\mathrm{J} / \mathrm{kg} \cdot \mathrm{K})$

$D \quad$ drive ratio

$d \quad$ internal diameter of the resonance tube (m)

$f_{0} \quad$ design operating frequency $(\mathrm{Hz})$

$h \quad$ height of cone volume (m)

$K \quad$ thermal conductivity $(\mathrm{W} / \mathrm{m} \cdot \mathrm{K})$

$k \quad$ wave number $\left(\mathrm{m}^{-1}\right)$

$L_{o} \quad$ length of the resonance tube (m)

$L_{p} \quad$ length of the resonance tube from $L_{o}(\mathrm{~m})$

$l \quad$ half of the thickness of the stack (m)

$L_{s} \quad$ stack length (m)

$L_{s n} \quad$ normalized stack length (dimensionless)

$P_{m} \quad$ mean pressure $\left(\mathrm{N} / \mathrm{m}^{2}\right)$

$P_{o} \quad$ pressure amplitude $\left(\mathrm{N} / \mathrm{m}^{2}\right)$

$Q_{c} \quad$ cooling power (W)

$Q_{c n} \quad$ normalized cooling power (dimensionless)

$R \quad$ gas constant $(\mathrm{J} / \mathrm{kg} \cdot \mathrm{K})$

$r \quad$ radius of cone volume (m)

$T_{m} \quad$ mean temperature (K)

$V_{b} \quad$ back volume $\left(\mathrm{m}^{3}\right)$

$V_{c} \quad$ cone volume $\left(\mathrm{m}^{3}\right)$

W acoustic power (W)

$W_{n} \quad$ normalized acoustic power (dimensionless)

$x_{s} \quad$ stack position (m)

$X_{s n} \quad$ normalized stack position (dimensionless)

$y_{o} \quad$ half of distance between the stack layers (m)

Greek Symbols

$\delta_{\kappa} \quad$ thermal penetration depth (m)

$\delta_{\kappa n} \quad$ normalized thermal penetration depth (dimensionless)

$\delta_{v} \quad$ viscous penetration depth (m)

$\Delta T_{m} \quad$ temperature difference across the two ends of stack (K)

$\Delta T_{m n} \quad$ normalized temperature difference across the two ends of stack (dimensionless)

$\gamma \quad$ ratio of isobaric to isochoric specific heat (dimensionless)

$\sigma \quad$ Prandtl number (dimensionless)

$\mu \quad$ viscosity $(\mathrm{kg} / \mathrm{m} \cdot \mathrm{s})$

$\rho \quad$ density $\left(\mathrm{kg} / \mathrm{m}^{3}\right)$

$\omega \quad$ angular frequency (rad/s)

\section{REFERENCES}

Akhavanbazaz, M., Siddiqui, M. H. K., and Bhat, R. B., 2007, "The Impact of Gas Blockage on the Performance of a Thermoacoustic Refrigerator," Experimental Thermal and Fluid Science, 32(1), 231-239.

http://dx.doi.org/10.1016/j.expthermflusci.2007.03.009

Assawamartbunlue, K., and Kanjanawadee, P., 2009, "Experimental Demonstration of Thermoacoustic Cooling," Journal of Research in Engineering and Technology, 6(1), 1-24.

Babaei, H., and Siddiqui, K., 2008, "Design and Optimization of Thermoacoustic Devices," Energy Conversion and Management, 49(12), 3585-3598.

http://dx.doi.org/10.1016/j.enconman.2008.07.002 
Bassem, M. M., Ueda, Y., and Akisawa, A., 2011, "Design and Construction of a Traveling Wave Thermoacoustic Refrigerator," International Journal of Refrigeration, 34(4), 1125-1131. http://dx.doi.org/10.1016/j.ijrefrig.2011.02.003

Beranek, L. L., 1954, Acoustics, New York, McGraw-Hill.

Berson, A., Michard, M., and Blanc-Benon, P., 2008, "Measurement of Acoustic Velocity in the Stack of a Thermoacoustic Refrigerator Using Particle Image Velocimetry," Heat and Mass Transfer, 44(8), 1015-1023.

http://dx.doi.org/10.1007/s00231-007-0316-x

Braun, J. E., Mongeau, L., Minner, B., Alexander, A., and Paek, I., 2001, "Evaluating the Performance of Thermoacoustic Cooling," Final Rep ort, Purdue University.

Hatazawa, M., 2006, "Oscillatory Flow in Thermoacoustic Sound Wave Generator," Journal of Thermal Science, 15(1), 92-96. http://dx.doi.org/10.1007/s11630-006-0092-9

Hofler, T. J., 1986, "Thermoacoustic Refrigerator Design and Performance," Ph.D thesis, University of California at San Diego.

Ke, H.-B., Liu, Y.-W., He, Y.-L., Wang, Y., and Huang, J., 2010, "Numerical Simulation and Parameter Optimization of ThermoAcoustic Refrigerator Driven at Large Amplitude," Cryogenics, 50(1), 28-35.

http://dx.doi.org/10.1016/j.cryogenics.2009.10.005

Nsofor, E. C., and Ali, A., 2009, "Experimental Study on the Performance of the Thermoacoustic Refrigerating System," Applied

Thermal Engineering, 29(13), 2672-2679.

http://dx.doi.org/10.1016/j.applthermaleng.2008.12.036

Nsofor, E. C., Celik, S., and Wang, X., 2007, "Experimental Study on the Heat Transfer at the Heat Exchanger of the Thermoacoustic Refrigerating System," Applied Thermal Engineering, 27(14-15), 2435-2442.

http://dx.doi.org/10.1016/i.applthermaleng.2007.03.008
Setiawan, I., Utomo, A. B. S., Nohtomi, M., and Katsuta, M., Year, "Experimental Study on Thermoacoustic Cooling System with Two Stacks in a Straight Resonator Tube," Proceedings of 10ème Congrès Français d'Acoustique, Ly on France.

Swift, G. W., 1988, "Thermoacoustic Engines," The Journal of the Acoustical Society of America, 84(4), 1145-1180. http://dx.doi.org/10.1002/9780470172520.ch61

Tang, K., Chen, G. B., Jin, T., Bao, R., Kong, B., and Qiu, L. M., 2005, "Influence of Resonance Tube Length on Performance of Thermoacoustically Driven Pulse Tube Refrigerator," Cryogenics, 45(3), 185-191. http://dx.doi.org/10.1016/j.cry ogenics.2004.10.002

Tasnim, S. H., Mahmud, S., and Fraser, R. A., 2011, "Measurement of Thermal Field at the Stack Extremities of a Standing Wave Thermoacoustic Heat Pump," Frontiers in Heat and Mass Transfer, 2(1), 1-10.

http://dx.doi.org/10.5098/hmt.v2.1.3006

Tijani, M. E. H., 2001, "Loudspeaker-Driven Thermo-Acoustic Refrigeration," Ph.D Thesis, Eindhoven University of Technology, Eindhoven.

Tijani, M. E. H., Zeegers, J. C. H., and de Waele, A. T. A. M., 2002, "Design of Thermoacoustic Refrigerators," Cryogenics, 42(1), 49-57. http://dx.doi.org/10.1016/S0011-2275(01)00179-5

Tu, Q., Gusev, V., Bruneau, M., Zhang, C., Zhao, L., and Guo, F., 2005, "Experimental and Theoretical Investigation on Frequency Characteristic of Loudspeaker-Driven Thermoacoustic Refrigerator," Cryogenics, 45(12), 739-746.

http://dx.doi.org/10.1016/j.cry ogenics.2005.09.004

Wetzel, M., and Herman, C., 1997, "Design Optimization of Thermoacoustic Refrigerators," International Journal of Refrigeration, 20(1), 3-21.

http://dx.doi.org/1212.1212121 